\title{
Co-culture of soil biofilm isolates enables the discovery of novel antibiotics
}

State Key Laboratory of Agricultural Microbiology, College of Resources and Environment, 
15

16

17

\section{Abstract}

Bacterial natural products (NPs) are considered to be a promising source of drug discovery. However, the biosynthesis gene clusters (BGCs) of NP are not often expressed, making it difficult to identify them. Recently, the study of biofilm community showed bacteria may gain competitive advantages by the secretion of antibiotics, implying a possible way to screen antibiotic by evaluating the social behavior of bacteria. In this study, we have described an efficient workflow for novel antibiotic discovery by employing the bacterial social interaction strategy with biofilm cultivation, co-culture, transcriptomic and genomic methods. We showed that a biofilm dominant species, i.e. Pseudomonas sp. G7, which was isolated from cultivated soil biofilm community, was highly competitive in four-species biofilm communities, as the synergistic combinations preferred to exclude this strain while the antagonistic combinations did not. Through the analysis of transcriptomic changes in four-species co-culture and the complete genome of Pseudomonas sp. G7, we finally discovered two novel non-ribosomal polypeptide synthetic (NRPS) BGCs, whose products were predicted to have seven and six amino acid components, respectively. Furthermore, we provide evidence showing that only when Pseudomonas sp. G7 was co-cultivated with at least two or three other bacterial species can these BGC genes be induced, suggesting that the co-culture of the soil biofilm isolates is critical to the discovery of novel antibiotics. As a conclusion, we set a model of applying microbial interaction to the discovery of new antibiotics. 
Keywords: non-ribosomal polypeptide synthase, inter-species interaction; multispecies

biofilm; Pseudomonas; soil bacteria;

37

\section{Importance}

Biofilm is an emergent form of bacterial life in natural settings. In a biofilm community, bacteria may employ several different strategies to avoid unwanted neighbors and gain competitive advantages, one of which is the secretion of antibiotics. These antibiotics are bacterial natural products and potential novel antimicrobial drug candidates. In this study, we have described an efficient workflow for novel antibiotic discovery by employing biofilm cultivation, co-culture and multiomics methods. Two novel NRPS-type BGCs were identified in a soil biofilm isolate, namely Pseudomonas sp. G7. Furthermore, the metabolite biodiversities of the two BGCs were analyzed by comparing them with those of related Pseudomonas spp. Additionally, we provide evidence showing that only when Pseudomonas sp. G7 was co-cultivated with at least two or three other bacterial species can these BGC genes be induced, suggesting that the co-culture of several bacterial isolates is critical to the discovery of novel antibiotics. 


\section{Introduction}

Biofilms are the most common mode of microbial existence in natural habitats (1). It has been estimated that in natural ecosystems, up to $99 \%$ of all bacterial activity is associated with bacteria that are attached to surfaces of and organized into biofilms (2). A biofilm mode-of-life not only gives cells several advantages (3) but also leads to intensive bacterial competition in multispecies biofilm communities (4). To gain an advantage in the competition, a bacterial species may rely on many different approaches, two of which are to inhibit the growth of other species by nutrient competition through the secretion of siderophores and to kill competitors by secreting broad-spectrum antibiotics (5). These approaches have been portrayed as the "pirate" and "killer" approaches, respectively (6). As microbial interactions trigger the production of antibiotics (7), it is logical to conclude that in a mature biofilm community, the dominant strain that is present is more likely to be a producer of antibiotics.

Since pathogen drug resistance is a major threat to human health, finding novel antibiotics is an urgent need. At present, natural products (NPs) are still the main sources of new antibiotics, which are generally secondary metabolites produced from a bacterial biosynthetic gene cluster (BGC) (8). With the revolutionary advances in genomics, metabolomics and other methodologies over last 10-20 years, it has become easy to identify BGCs in a known bacterial genome or meta-genome using bioinformatic tools and resolve the structures of new biomolecules through high-pressure liquid chromatography (HPLC), 
mass spectrometry and nuclear magnetic resonance (NMR) spectroscopy (9). However,

75 genomic analysis has revealed that many microorganisms have a far greater potential to produce specialized metabolites than is suggested by classical bioactivity screens, as many BGCs in the genome are silent and therefore not expressed under standard laboratory growth conditions (10). This lack of expression makes the screening for novel antibiotic biosynthesis in a bacterial strain a limitation in such a process.

To overcome the limitations in screening and/or try to activate the expression of BGCs, multiple methods have been developed (10). For example, iChip was used to simultaneously isolate and grow soil bacteria under in situ conditions (11), which not only greatly increases the throughput of the screening but also enables the acquisition of a large number of bacteria and their products that cannot be cultured using conventional media. In addition, some BGCs are imported into engineered model bacteria and heterologously expressed under strong promoters to promote NP biosynthesis (12). Notably, these efforts are highly dependent on bacterial monocultures while ignoring the essential role of interspecies interactions on inducing BGC gene expression.

Strikingly, methods of co-cultivation, which were initially used to study interspecies interactions in biofilms, are beginning to be applied to the discovery of new antibiotics (7, 13). For example, co-culturing the fungus Aspergillus nidulans and 58 actinomycetes species can activate a variety of silent gene clusters in A. nidulans, such as polyketide orsellinic acid, lecanoric acid, and the cathepsin K inhibitors F-9775A and F-9775B (14). 
The coculture of marine invertebrate-associated bacteria has enabled the discovery of a new

95 likely to synthesize antibiotics. Therefore, we constructed a model system to produce

113 biofilms of bacteria derived from paddy soil. During cultivation, the community structure of

antibiotic, keyicin (15).

In the current study, we first cultivated soil biofilms using a model system and investigated the microbial succession of biofilm communities during a seven-day cultivation period. From the soil biofilms, twenty-four bacterial strains were isolated. In this case, all of the isolates were potentially dominant species in the biofilm community. Seven isolates belonging to different genera were subsequently picked, and intergenus interactions between the isolates were assessed using a co-culture and the Nunc-TSP Lid system. We found that the presence of Pseudomonas isolate G7 had a significant effect in determining whether synergy or antagonism occurred in the four-species biofilm, as the synergistic combinations preferred to exclude this strain while the antagonistic combinations did not. Furthermore, de novo (meta-)transcriptomic analysis revealed that novel non-ribosomal polypeptide synthase (NRPS) genes were significantly upregulated in a G7-containing four-species co-culture, which led to the discovery of two novel antibiotic biosynthetic NRPS BGCs.

\section{Results}

\section{Workflow of this study}

We hypothesized that the dominant species in the biofilm communities were more che 
114 the biofilm was monitored by 16S rRNA gene sequencing. After 7 days of cultivation, the

115 biofilm community was stabilized (see below), and cultivatable bacteria were isolated from

116 the mature biofilm. These isolates are believed be highly competitive in the formation of

117 biofilm, and the isolation of these bacteria allowed us to check the synergistic and

118 antagonistic effects on biofilm formation through the use of co-cultures and high-throughput

119 crystal violet assays (Fig. 1), ultimately identifying the most competitive members.

Bacterial isolates were obtained from biofilm cultivated from a paddy soil microbial

community

Biofilm cultivation was initiated from soil microbial communities which had been

extracted from the top layer of rice paddy soil, as described in the experimental procedures.

124 During cultivation, the succession of the bacterial community in the biofilm was

125 investigated using $16 \mathrm{~S}$ rRNA sequencing. The experiment was replicated twice and sampled

126 at 1,2, 4 and 7 days of cultivation using an autoclaved soil solution as the only source of

127 nutrients. As a result, 269,439 valid sequences of the V4-V5 region of bacterial 16S rRNA

128 genes were produced. In these samples, 256 operational taxonomic units (OTUs), defined

129 by $97 \%$ sequence similarity, were classified into 11 phyla, including Firmicutes,

130 Proteobacteria, Bacteroidetes, and Verrucomicrobia (Fig. 2A). The most abundant phylum

131 was Firmicutes and was mainly represented by three genera, namely, Bacillus, Lactococcus,

132 and Enterococcus. The second most abundant phylum was Proteobacteria and was mainly

133 represented by the genera Pseudomonas, Massilia, and Pseudogulbenkiania. 
144 dramatically, with the proportion changing from $\sim 80$ to $\sim 20 \%$ of all sequences. In contrast,

145 the abundance of Proteobacteria increased from $\sim 15$ to $65 \%$ (Fig. 1C), indicating that the

146 dominant Firmicutes species at day 1 were replaced by the Proteobacteria species in

147 biofilms.

Subsequently, twenty-four bacterial strains were isolated from the biofilm communities

149 on day 7 (Table S1). As expected, all of the strains except Microbacterium sp. D12 were

150 from dominant phyla in the biofilms, including twelve genera of Proteobacteria and three

151 genera of Bacteroidetes (Fig. 3). These strains were well separated in the phylogenetic tree

152 (Fig. 3), and their coexistence in biofilms suggests that interspecies interactions are

153 ubiquitous among the bacteria. 


\section{in multispecies biofilm formation}

As stated above, the interactions between different species in biofilm may lead to the

(Fig. 3) for further analysis. These strains were chosen, in part, because our isolates have relatively rich diversity of species and we expect that more distantly related species may be

164 more likely to compete with each other. These isolates were mixed into four-species

165 co-cultures, and the effects on biofilm formation were analyzed using the crystal violet

166 assay. According to previous data (16), the synergistic or antagonistic effects of co-culture

167 on biofilm formation can be determined by comparing the biofilm biomasses of the

168 co-culture with the monocultures of contained species. That is, when the biofilm biomass of

169 the co-culture is significantly higher than the highest single-species monoculture, the

170 co-culture promotes biofilm formation. When the biofilm biomass of the co-culture is

171 significantly less than the lowest single-species monoculture, the co-culture inhibits biofilm

172 formation.

173 The biofilm formation of monocultures was initially determined, and the results showed 
174 that monocultures biofilm formation varied greatly (Fig. S2), with the biofilm formation of

175 Pseudomonas sp. G7 being the highest. Furthermore, a total of 35 different four-species

176 consortia were screened, the biofilm biomasses of twelve combinations were greater than

177 the highest single-species biofilm biomass (Fig. 4). According to the assumptions described

178 above, this result suggested that synergistic effects on biofilm formation exist in the twelve

179 combinations. Of the other 23 combinations, sixteen showed no significant changes while

180 seven showed antagonistic effects (Table S2). Interestingly, most of the synergistic

181 combinations (11 of 12) did not include the strain Pseudomonas sp. G7 (Fig. 4). However,

182 most of the antagonistic combinations (6 of 7) did have this strain (Fig. 4, and Table S2).

183 Fisher's exact test showed that the presence of Pseudomonas sp. G7 in co-culture was the

184 most significant factor in determining the fate of biofilm formation $(\mathrm{P}=0.001)$, followed by

185 Acinetobacter sp. G18 $(\mathrm{P}=0.006)$. All the other species were not significant $(\mathrm{P}>0.05)$.

186 Consistently, we further found that Pseudomonas sp. G7 can form a zone of inhibition on

187 lawns of Aquitalea sp. G2 and Flavobacterium sp. G16 (Fig. S3B and E), and the

188 Acinetobacter sp. G18 can form zones of inhibition on lawns of Aquitalea sp. G2 (Fig. S3B),

189 indicating that the two species may be promising antibiotic producers.

190 De novo meta-transcriptomic analysis revealed the induction of NRPS antibiotic

191 synthase genes in Pseudomonas sp. G7 during four-species co-culture

192 We chose Pseudomonas sp. G7 for further confirmation of its capacity to produce

193 antibiotics. To study the molecular mechanisms of Pseudomonas sp. G7 in competition with 
194 other species, we compared the transcriptomic profiles of a four species co-culture of

195 Novosphingobium sp. G5, Pseudomonas sp. G7, Flavobacterium sp. G16 and

196 Microbacterium sp. D12 and monocultures of each individual species using high-throughput

197 mRNA sequencing technology (RNA-seq). Notably, our approach is slightly different from

198 more conventional ones. In the construction of the library, we did not build separate

199 libraries for four mono-cultures but chose to pool them together and construct a single

200 control library (CK). In this way, we only needed to build two transcriptome libraries

201 (without replicates). After sequencing, we first de novo assembled the reads, then separated

202 the sequences into the four species using the meta-genomic binning method. Given that we

203 know the species composition in the co-culture system, the use of taxonomic barcodes or

204 phylogenetic marker genes can achieve satisfactory accuracy.

After de novo assembly of sequencing reads and meta-genome binning, we finally

206 obtained 4060 unigenes: 1463 unigenes from Novosphingobium sp. G5 (3.5 M bps in total),

2071280 unigenes from Pseudomonas sp. G7 (7.0 M bps in total), 1033 unigenes from

208 Flavobacterium sp. G16 (0.14 M in total), and 284 unigenes from Microbacterium sp. D12

209 ( $0.52 \mathrm{M}$ in total). The variances between the cumulative sizes of the unigenes are attributed

210 to the different abundances of the RNA-seq reads of each species. For example,

211 approximately $95.34 \%$ of the total co-culture reads aligned to Pseudomonas sp. G7, while

212 only $0.01 \%$ of the reads aligned to Flavobacterium sp. G16. Due to the lack of RNA-seq

213 reads, the completion of the genome assembly of the other three species was limited. 
214 However, this lack of reads from other species also showed that Pseudomonas sp. G7 was

215 absolutely dominant in the co-culture.

216 Most of the unigenes have multiple open reading frames and polycistronic structures.

217 Therefore, genes encoded by these unigenes were predicted prior to the analysis of their

218 expression, as described in the methods section. More than twelve thousand genes were

219 characterized and annotated, and the expression of 461 genes was subsequently found to be

220 significantly changed in the four-species co-cultures compared to their monoculture values

221 (Fig. 5A). The gene expression of Pseudomonas sp. G7 was the most affected (421 genes

222 were differentially expressed), followed by those of Novosphingobium sp. G5 (37 genes)

223 and Microbacterium sp. D12 (3 genes) (Fig. 5). No significant changes in gene expression

224 were found in Flavobacterium sp. G16. Of the differentially expressed genes, 383 were

225 successfully annotated by Clusters of Orthologous Groups (COGs), with the number of

226 genes in each functional category being shown in Fig. 5B, grouped by different species.

As shown in Fig. 5B, most of the genes in the COG category of ribosomal structure

228 (COG: J) were downregulated in both Pseudomonas sp. G7 and Novosphingobium sp. G5,

229 suggesting that protein translation was inhibited in co-culture. Additionally, multiple

230 metabolic genes (COG: C, G, E, F, H, I, and P) of both species were also significantly

231 changed. In Pseudomonas sp. G7, twenty-one transcriptional regulators (COG: K) and

232 twenty-nine signal transduction genes (COG: T) were differentially expressed, implying a

233 complex regulatory mechanism involved in maintaining the activity of the species when in 
234 the competition with the other three species. In addition, forty-five genes related to cell

235 motility (COG: N) were differentially expressed in Pseudomonas sp. G7, with the majority

236 of these genes, which encode flagellar basal proteins and/or are involved in flagellar

237 biosynthesis pathways, being downregulated. This downregulation suggests that cell

238 motility was inhibited, and biofilm formation was enhanced in co-culture.

239 Notably, twenty-four genes involved in secondary metabolite biosynthesis, transport

240 and catabolism (COG: Q) were differentially expressed (Fig. 5B), with the majority of

241 non-ribosomal peptide synthetase (NRPS) encoding genes being upregulated more than

242 twenty times in co-culture (Table 1). These NRPS genes were located in five unigenes (Fig.

243 S4). Sequence analysis of these unigenes revealed that they all showed a high sequence

244 similarity (but importantly, were not identical) to a previously identified bananamide

245 biosynthesis gene cluster in Pseudomonas sp. DR 5-09 (17). Except for a gap of

246 approximately $1 \mathrm{~kb}$ in the banA gene, the entire cluster was covered by six unigenes of

247 Pseudomonas sp. G7 (Fig. S4). We thus identified that a NRPS antibiotic BGC is present in

$248 \quad$ Pseudomonas sp. G7 and highly expressed in co-culture.

249 The whole-genome sequencing of Pseudomonas sp. G7 revealed two novel

250 NRPS-antibiotic BGCs

251 Although the meta-transcriptomic analysis showed the presence of a novel NRPS BGC

252 in Pseudomonas sp. G7, the sequence of the BGC was incomplete, and therefore, we further

253 performed whole-genome sequencing of this soil biofilm isolate. The complete genome of 
Pseudomonas sp. G7 is 6,336,169 bp, with a GC content of $60.42 \%$, and encodes 5855

genes. The NRPS BGC, which is similar to the bananamide BGC, was then identified by antiSMASH as described in the methods section. Its core region is approximately $35 \mathrm{~kb}$ and

by antiSMASH, the two NRPS BGC are both synthetic antibiotics that have not been

respectively.

Paddyleftmide was predicted to consist of seven amino acid residues of (d-leu-asp) +

264 (thr-d-val-leu) + (d-leu-ile) (Fig. 6C). The first two amino acids were added by

265 C6Y56_11440, the second three amino acids are added by C6Y56_11445, and the last two

266 amino acids are added by C6Y56_11450 (Fig. 6A). Likewise, paddyrightmide was

267 predicted to have six amino acid residues of (arg-pro-cys) $+($ nrp $)+($ val-pro $)$ (Fig. 6D). The

268 first three amino acids were added by C6Y56_11675, the second amino acid were added by

269 C6Y56_11680, and the last two amino acids were added by C6Y56_11685. Notably, both

270 paddyleftmide and paddyrightmide are chiral compounds, and their real structures may be

271 quite different from this in silico prediction.

Although we found that the two newly discovered antibiotic BGCs were not included 
274 publicly available genomes at the sequence level. As shown in Table 2, all of the related

275 genomes were Pseudomonas species. Among the genomes was the Pseudomonas

276 fluorescens Pf0-1 strain, which is a model microorganism for P. fluorescens studies (19). In

277 addition, $P$. fluorescens strain BW11P2 was a relatively early isolate of Pseudomonas (20);

278 however, its ability to synthesize bananamide was only recently identified (17). In addition,

279 several other strains were isolated in recent years. All of these bacteria were described as

280 being isolated from soil samples, except for Pseudomonas moraviensis strain BS3668,

281 which did not have information available.

282 We compared the phylogenetic similarities of paddyleftmide and paddyrightmide

283 BGCs with similar BGCs in other Pseudomonas species. In this analysis, the amino acids of

284 the core biosynthetic genes were used in a multilocus sequence analysis as described in the

285 methods section. The paddyleftmide BGC was phylogenetically different from all other

286 analogs and was not assigned to an end clade of the evolutionary tree (Fig. 7A). However,

287 the distance matrix clearly showed that the differences between Pseudomonas sp. G7, P.

288 fluorescens Pf0-1 and P. moraviensis strain BS3668 is much greater than the differences

289 between Pseudomonas sp. G7, P. fluorescens strain BW11P2, Pseudomonas sp. MS586 and

290 Pseudomonas sp. DR 5-09 (Fig. 7B). Likewise, the paddyrightmide BGC is quite unique as

291 well (Fig. 7C). The distance between the BGC and all other BGC analogs is greater than the

292 distance between any of the other analogs (Fig. 7D). However, the absolute difference is

293 more significant for the paddyleftmide BGC, as can be seen when the distance matrixes 
294 were colored with an identical color palette (Fig. 7B and 7D). As a conclusion, the two

295 BGCs found in Pseudomonas sp. G7 are novel antibiotic producing elements, reflecting the

296 biological diversity of the secondary metabolites of Pseudomonas spp.

297 Reverse verification of the effects of co-culture promoting antibiotic biosynthesis using

298 qRT-PCR

299 After obtaining the whole-genome sequence of Pseudomonas sp. G7, it is easy to find

300 that the upregulated NRPS genes in the Meta-transcriptomic analysis are all from

301 paddyleftmide and paddyrightmide core biosynthetic genes (Table 1). Although we came to

302 the above conclusions through meta-transcriptomic analysis and further whole-genome

303 sequencing, it is still doubtful whether this process is a coincidence; that is, if we choose

304 different combinations of co-cultures, can we still get the same results?

305 To answer this question, we used qRT-PCR to detect multiple co-culture systems

306 containing Pseudomonas sp. G7. The results of the experiments finally demonstrated that

307 even if different co-culture combinations were chosen, we could still find that the NRPS

308 gene was induced by the species-species interactions. Fig. 8A shows the gene expression of

309 the paddyleftmide synthetic gene (C6Y56_11440) in the Pseudomonas sp. G7 mono-culture,

310 four two-species co-cultures, six three-species co-cultures, and four four-species co-cultures

311 (all the co-cultures contain Pseudomonas sp. G7). Compared with monoculture, the

312 expression of the paddyleftmide synthetic gene was downregulated in all the two-species

313 co-cultures but was mostly upregulated in the three-species co-cultures and was upregulated 
314 in all the four-species co-cultures. In general, it can be found that the co-cultures containing

315 at least two species effectively induce paddyleftmide biosynthetic gene expression, and the

316 more species the co-culture contains, the more obvious the upregulation is. Similar

317 conclusions were obtained when measuring the gene expression of the paddyrightmide

318 biosynthetic gene (C6Y56_11675, Fig. 8B). Although there are differences in the expression

319 of the antibiotic synthetic genes in different four species of co-cultures, paddyleftmide and

320 paddyrightmide will eventually be identified in Pseudomonas sp. G7, regardless of the

321 combination of species we chose in the four species of co-culture. This result reversely

322 validates that our workflow is robust.

323

324

325

326

\section{Discussion}

This study describes a workflow for screening novel antibiotics from soil using biofilm cultivation, mixed co-cultures, and meta-omics techniques. Following this workflow, we demonstrate its effectiveness with the discovery of two novel antibiotic BGCs, which are named paddyleftmide and paddyrightmide, from a paddy soil community.

We used flow cells to culture the biofilm of soil microbial communities. The species present in these biofilms were all derived from soil microbial communities, but due to the heterogeneity of the soil, these microorganisms may not have reflected in the natural environment. Despite the high biodiversity and biomass of living bacteria in soils, only approximately $10^{-6} \%$ of the soil surface is covered by microorganisms (21). Therefore, soil bacteria can only contact their close neighbors. The estimated interaction distances of most 
334 soil microbes occur over distances no greater than a few tens of micrometers (22). To allow

335 these bacteria to contact and compete with each other, it is necessary to artificially mix them

336 and grow them together. This can be achieved through the application of in vitro model

337 systems where an environmental sample is used as inoculum, or to isolate biofilm-forming

338 strains from specific environments for use in co-cultures $(23,24)$.

By mixing the bacterial species together and fostering competition between species,

340 highly competitive species can be isolated, which will more likely be producers of

341 antibiotics. The 256 OTUs identified in the biofilm samples are at a comparable level to

342 those of a microscale single bacterial community in bulk soil, which is, on average, limited

343 to fewer than 100 species and tens of hundreds of microbial cells (25). However, this

344 number of OTUs is much smaller than that of soil microbiomes, whose total OTUs may

345 range from 3,731 to 27,147 (26-28). With such a biofilm cultivation process, highly

346 competitive species are enriched for in the biofilm community, and therefore, the efficiency

347 of subsequent screens can be improved.

348 Soil is believed to be the most complex biomaterial (21) and have the most vast

349 microbial biomass and biological diversity (29) on the planet. About two-thirds of all

350 naturally derived antibiotics currently in clinical use, as well as many anticancer,

351 anthelmintic, and antifungal compounds, are produced mostly by the soil-dwelling

352 Actinobacteria, in particular by species of Streptomycetes (30). However, in our biofilm

353 community, the Actinobacteria were not the dominant species. This may be due to culture 
conditions, since we used autoclaved soil water as the only source of nutrients and

cultivated biofilms in an open flow model system. The resulting conditions for biofilm

cultivation are aquatic, aerobic, and have a low supply of nutrients, which are not conditions

Recent studies have shown that microorganisms in the soil are different from those in

antibiotics, it will significantly affect the formation of biofilm in multispecies co-cultures.

366 Of the 20 combinations containing Pseudomonas sp. G7, only one biofilm biomass

367 increased when compared to the monocultures, and of the 7 microbial combinations that

368 lowered biofilm biomass, 6 contained the isolate (Fig. 4, and Table S2). Most of the

369 synergistic combinations (11 out of 12) did not include the Pseudomonas sp. G7 strain, and

370 most of the antagonistic combinations (6 out of 7) had this strain (Fig. 4, and Table S2). The

371 presence or absence of Pseudomonas sp. G7 significantly influenced the biomass of

372 co-cultured multispecies biofilm $(\mathrm{P}=0.001)$. At the same time, the presence of

373 Acinetobacter sp. G18 also has a significant effect on the formation of multispecies biofilm 
$(\mathrm{P}=0.006)$. Consistently, both of these bacteria form zones of inhibition when grown on the

377 unknown antibiotic BGC. This evidence showed that it is feasible to screen for

biofilm.

We believe that the key to the discovery of new antibiotics is to stimulate their

biological activity. If these antibiotics are only encoded by microbial genome but not

expressed, no antibiotic will be produced, and the difficulty in screening for these

compounds will be enormous. This article presents a method to elicit the biological activity

384 of antibiotics that are capable of being synthesized by microorganisms as well as a method

385 of co-cultivation to identify these antibiotic-producing bacteria. Notably, based on our

386 results, the co-culture may need at least three species to achieve sufficient stimulation to

387 elicit antibiotic gene expression (Fig. 8). Co-cultivation and the measurement of biofilm

388 biomass with crystal violet staining are low-cost, scalable methods (Fig. 1).

389 High-throughput screening can easily be achieved using instruments such as multiwell

390 microplates, loading robots, and microplate readers $(34,35)$.

When using conventional methods to study gene expression changes in co-culture systems, a reference genome of each species present in the co-culture is required and many mono-culture transcriptomic libraries are constructed as controls (36). By comparing the 
394 transcriptomes of mono-cultures and co-cultures, differentially expressed genes can be

395 identified. In this context, identifying gene expression changes in a four-species co-culture

396 may require the genomic sequencing of four species, four separate mono-culture

397 transcriptome sequences, and one transcriptome sequence of the co-culture. After

398 sequencing, the bioinformatics pipeline for transcriptomic analysis with reference will be

399 utilized and the expression changes in the co-culture system can be obtained. This process is,

400 obviously, very costly. Some other methods, such as designing species-specific microarray

401 chips (37), or separating the species present in co-culture by molecular markers (38), also

402 have the same shortage and are not applicable for novel bacterial isolates without a

403 preexisting knowledge of their genome. In this study, we used an alternative method of

404 meta-transcriptome sequencing by combining the four mono-cultures together to construct a

405 single transcriptome library (control). The control library was then sequenced in parallel

406 with the transcriptome library of the four-species co-culture, followed by de novo assembly

407 of meta-transcripts. By genome binning, the assembled contigs can be assigned to 4

408 different species. Then, the gene expression differences in each species can be determined.

409 In this framework, it is only necessary to construct two transcriptome libraries, one for

410 mixed mono-cultures of the controls and the other for the co-culture, decreasing the overall

411 sequencing cost by more than 4 times. Notably, we can still identify the upregulation of

412 antibiotic BGC genes and determine the species in whose genome the BGC is located.

413 These results showed that this improvement of cost does not substantially effect the 
414 efficiency of the screening and is therefore beneficial for large-scale screening.

417 beneficial to improve the accuracy of genome binning (because different families of

418 microorganisms were mixed in the co-culture) and to the subsequent preservation and

419 genetic modification of the strains. However, this may also lead to the loss of some potentially non-cultivable microorganisms who may secrete antibiotics in biofilms.

\section{$421 \quad$ Materials and Methods}

\section{Soil and strains}

Soil samples were collected from two paddy fields that are located in Dajin Town,

424 Wuxue City, Hubei province, China $\left(115^{\circ} 33^{\prime} \mathrm{E}, 2^{\circ} 51^{\prime} \mathrm{N}\right)$. This area has a subtropical

425 monsoon climate. In each paddy, five samples were taken from the top layer of soil, which

426 is approximately $5-15 \mathrm{~cm}$ deep, and thoroughly mixed. After they were sampled, soils were

427 partially air-dried and passed through a $2 \mathrm{~mm}$ sieve for use in subsequent experiments. The

428 strains used in this study (Table S1) were all isolated from cultivated biofilm communities

429 using a previously described method (23). The 16S rRNA genes of these strains were

430 amplified with primers 27F and 1492R and sequenced using an ABI 3730XL DNA Analyzer

431 (Applied Biosystems, US). The sequences were assembled by ContigExpress software

432 (Invitrogen) and submitted to SINA Alignment Service to obtain their taxonomic 433 classifications (39). 


\section{Biofilm cultivation in a parallel flow system}

The system was set up as previously described (23). For each sample, sieved soil (150 g,

New Jersey, USA) several times and left until the agar cover solidified. The glass slides

were then placed in a rack attached to a beaker containing soil slurry so that the slides were

covered by the liquid phase of the slurry but did not disturb the solid. The beaker was

444 the agarose gels on the glass plates (i.e., biofilms) were collected into a tube. Then, $300 \mu \mathrm{L}$

445 of sterilized glycerol was added to the tube, the biofilms were thoroughly mixed and stored

446 at $-80{ }^{\circ} \mathrm{C}$ to be used as a starter inoculum. The starter inoculum was inoculated onto the

447 surface of seven agar-covered glass slides, which were then assembled between two holders

448 in a homemade plexiglass flow chamber and left for $4 \mathrm{~h}$ to allow the bacteria to attach to the

449 agar. After bacterial attachment, $\sim 300 \mathrm{~mL}$ of autoclaved soil water (ASW) was circulated

450 through silicone tubes over the slides in a closed circuit with a flow rate of $7.5 \mathrm{~mL} / \mathrm{min}$ at

451 room temperature for 7 days. Biofilms on glass slides were harvested at days 1, 2, 4 and 7

452 by removing one glass slide from the flow chamber. Slides with attached biofilms were

453 rinsed twice with $0.9 \% \mathrm{NaCl}$, and biofilms were collected into a tube and divided equally 
into two parts. One part was subjected to $16 \mathrm{~S}$ rRNA gene sequencing, and the other was used for strain isolation.

\section{DNA extraction and high-throughput 16S rRNA gene sequencing}

5'-CCGTCAATTCMTTTRAGTTT-3', where barcode is an eight-base sequence unique to

464 each sample. PCR reactions were performed in triplicate in a $20 \mu \mathrm{L}$ mixture containing $4 \mu 1$ 465 of $5 \times$ FastPfu Buffer, $2 \mu \mathrm{L}$ of $2.5 \mathrm{mM}$ dNTPs, $0.8 \mu \mathrm{L}$ of each primer $(5 \mu \mathrm{M}), 0.4 \mu \mathrm{l}$ of

466 FastPfu Polymerase, and 10 ng of template DNA. Amplicons were extracted from $2 \%$

467 agarose gels and purified using an AxyPrep DNA Gel Extraction Kit (Axygen Biosciences,

468 Union City, CA, U.S.) according to the manufacturer's instructions and were quantified

469 using a QuantiFluor ${ }^{\mathrm{TM}}$-ST (Promega, U.S.). Purified amplicons were pooled to be

470 equimolar and paired-end sequenced $(2 \times 250)$ on an Illumina MiSeq platform according to

471 standard protocols. Raw FASTQ files were demultiplexed and quality-filtered using QIIME

472 (version 1.9.1) with the following criteria: (i) The 300 bp reads were truncated at any site

473 receiving an average quality score $<20$ over a 50 bp sliding window, and any reads shorter 
474 than $50 \mathrm{bp}$ were also truncated. (ii) Exact barcode matching was required, a two nucleotide

475 mismatch was allowed in primer matching, and reads containing ambiguous characters were

476 removed. (iii) Sequences that only overlapped more than $10 \mathrm{bp}$ were assembled according

477 to their overlap sequence. Reads that could not be assembled were discarded. Operational

478 Taxonomic Units (OTUs) were clustered with 97\% similarity cutoff using UPARSE

479 (version $7.1 \mathrm{http}: / /$ drive5.com/uparse/), and chimeric sequences were identified and

480 removed using UCHIME. The taxonomy of each $16 \mathrm{~S}$ rRNA gene sequence was analyzed by

481 RDP Classifier (http://rdp.cme.msu.edu/) against the Silva (SSU123)16S rRNA database

482 using a confidence threshold of $70 \%$ (40). Principal component analysis and temporal

483 analysis were performed by STAMP software (version 2.1.3) (41). During temporal analysis

484 of soil biofilms, an analysis of variance (ANOVA) test was employed, and the most

485 significantly changed bacterial groups were identified with p-values $<0.05$. The raw reads

486 of $16 \mathrm{~S}$ rRNA gene sequencing were deposited into SRA database

487 (https://trace.ncbi.nlm.nih.gov/Traces/sra/sra.cgi?) under the BioProject of PRJNA420805.

\section{Crystal violet assay}

A modified version of the crystal violet (CV) assay for the detection of biofilms using

490 96-well cell culture plates was applied as previously described (42). Biofilms formed on

491 pegs of the Nunc-TSP lid system were measured after $24 \mathrm{~h}$ of incubation unless otherwise

492 indicated. To wash off planktonic cells, the peg lid was successively transferred to three

493 microtiter plates containing $200 \mu \mathrm{L}$ of distilled deionized water per well. After the biofilms 
were stained for 20 min with $180 \mu \mathrm{L}$ of a $0.1 \%(w / v)$ aqueous $\mathrm{CV}$ solution, the lid was again

a statistically significant difference.

501

502 sequenced by HiSeq 2500 platform using standard protocols (BGI, China). After filtering, 512 the remaining clean reads were de novo assembled into transcripts using Trinity (version

\section{Meta-transcriptomic analysis}

$$
\text { Exponential-phase cultures of Novosphingobium sp. G5, Pseudomonas sp. G7, }
$$

Flavobacterium sp. G16 and Microbacterium sp. D12 were adjusted to an optical density of 0.15 at $600 \mathrm{~nm}$ in tryptic soy broth (TSB) medium. One milliliter of each culture and a mixture of containing $250 \mu \mathrm{L}$ of each individual culture, were then inoculated into $50 \mathrm{~mL}$ of fresh TSB medium. After $12 \mathrm{~h}$ of incubation at $25{ }^{\circ} \mathrm{C}$ with shaking (200 rpm), cells were collected by centrifugation at $4000 \times \mathrm{g}$. Then, equal amounts of monoculture cells were mixed and treated as a monoculture control (CK). Total RNA of CK and the four-species co-culture were extracted with TRIzol reagent (Invitrogen) following the manufacturer's instructions. RNA-seq libraries constructed with co-culture RNA and the control RNA were 2.0.6) (43), followed by gene family clustering with Tgicl (version 2.0.6) (44) to get final 
514 unigenes. Binning of unigenes was performed by analyzing the taxonomic classification of

515 BLAST hits in MEGAN (version 6.4.15) under the family level (45), as the four species in

516 the co-culture are from four different families. MetaGeneMark (version 3.25) was employed

517 to predict the putative genes in those unigenes (46). Reads were then separately mapped to

518 the predicted genes of each species using bowtie2, differentially expressed genes were

519 characterized by RSEM-EBSeq pipeline (version 1.2.22) (47, 48). This pipeline is capable

520 of obtaining high confidence results in the absence of experimental replicates, and thus is

521 very suitable for high-throughput screening applications. COG annotation of genes was

522 carried out by aligning them to the COG database using BLAST (access Jan, 2017) (49).

523 Raw sequence data were deposited in the SRA database

524 (https://trace.ncbi.nlm.nih.gov/Traces/sra/sra.cgi?) under the BioProject of PRJNA401492.

525 Whole genome sequencing of Pseudomonas sp. G7

526 The Pseudomonas sp. G7 strain genome was sequenced using a PacBio RS II platform

527 and Illumina HiSeq 4000 platform at the Beijing Genomics Institute (BGI, Shenzhen,

528 China). Four SMRT cells were used by the PacBio platform to generate the subreads set.

529 PacBio subreads with length less than $1 \mathrm{~kb}$ were removed. The program Pbdagcon

530 (https://github.com/PacificBiosciences/pbdagcon, accessed Feb, 2018) was used for

531 selfcorrection. Draft genomic unitigs, which are uncontested groups of fragments, were

532 assembled using the Celera Assembler against a highquality corrected circular consensus

533 sequence subreads set. To improve the accuracy of the genome sequences, GATK 
534 (https://www.broadinstitute.org/gatk/, accessed Feb, 2018) and SOAP tool packages

535 (SOAP2, SOAPsnp, SOAPindel, accessed Feb, 2018) were used to make single-base

536 corrections. To trace the presence of any plasmid, the filtered Illumina reads were mapped

537 using SOAP to the bacterial plasmid database (http://www.ebi.ac.uk/genomes/plasmid.html,

538 accessed July 8, 2016). The complete genome sequence of Psuedomonas sp. G7 was

539 submitted to NCBI Genbank database and annotated by Prokaryotic Genome Annoation

540 Pipeline (accessed Mar 9, 2018) (50). To reveal the antibiotic biosynthesis gene clusters in

541 the strain, the sequence was analyzed by antibiotics and secondary metabolite analysis shell

542 (antiSMASH, version 4.0) (9), followed by manually correction.

\section{Comparative genomics}

544 We used comparative genomics to analyze the sequence diversity of the two antibiotic

545 synthesis BGCs found in Pseudomonas sp. G7. Briefly, the nucleotide sequences of two

546 NPRS antibiotic BGCs were used to query the non-redundant nucleotide collection (nt

547 database, accessed Apr 4, 2018) for any known similar BGCs by NCBI BLAST. As a result,

548 five Pseudomonas spp. were found have an architecture similar to that of the two BGCs

549 (Table 2). The genomes of the five species were then analyzed by antiSMASH (9), and the

550 corresponding BGCs were identified. To compare BGCs, the protein sequences of their

551 encoded genes were aligned and the phylogenic distance calculated by msa package

552 (version 1.12.0) (51), then a phylogenetic tree was constructed using a maximum likelihood

553 method with ape package (version 5.1) (52). Trees and distances were visualized using 
554 ggtree (version 1.12.0)

and

pheatmap

(version

1.0 .8

555 https://CRAN.R-project.org/package=pheatmap), respectively.

RNA extraction, cDNA synthesis and Quantitative real-time PCR (qRT-PCR)

557 The experiments were carried out as previously described (54). Briefly, RNA was isolated

558 from $1 \mathrm{~mL}$ of broth cultures with TRIzol Reagent (Invitrogen) following the manufacturer's

559 instructions. For reverse-transcription PCR, the extracted RNA was first digested by gDNA

560 wiper (included in the kit) to remove possible genomic DNA contamination and then used

561 as a template for synthesis of cDNA using 6-mer random primers and the HiScript II 1st

562 Strand cDNA Synthesis Kit (Vazyme). The cDNA was used to amplify the core synthetic

563 genes of the NRPS BGC and the 16S rRNA gene of Pseudomonas sp. G7 using their

564 specific primers with AceQ ${ }^{\circledR}$ qPCR SYBR ${ }^{\circledR}$ Green Master Mix (Vazyme) and the standard

565 SYBR protocol in a Quantstudio 6 Flex instrument (Applied Biosystems). For each PCR,

566 the experiments were performed in at least triplicate and the amplifications were checked by

567 navigating the amplification curve and melting curve. Gene expression was normalized to

568 the levels of 16S rRNA and the changes in gene expression were calculated using the $2^{-\Delta \Delta \mathrm{Ct}}$

569 method (55).

$570 \quad$ Data accessibility

$571 \quad 16 \mathrm{~S}$ rRNA gene sequences of soil biofilm isolates: Genbank accession

572 MF689010-MF689033. 16S rRNA gene amplicon sequencing reads were submitted to SRA

573 database under the BioProject of PRJNA420805. Meta-transcriptome sequencing reads 
574 were submitted to SRA database under the BioProject of PRJNA401492. Whole genome

575 sequencing results were submitted to Genbank under the accession of CP027561.

\section{Statistics analysis}

In addition to the above described statistical analysis with specific software, some other

578 statistical analysis was performed with R (https://www.r-project.org/).

\section{Conflicts of interest}

The authors declared there is no conflict of interest.

\section{Acknowledgements}

This work was supported by the National Key R\&D Program of China

583 (2016YFD0800206), the National Natural Science Foundation of China (41522106). The

584 authors would like to thank Dr. Qian Wang, currently works at Montana State University,

585 for her inspirational comments on this study.

\section{Author Contributions}

587 P.C. conceived the topic. C.H.G. and Z.L. performed experiments, C.H.G., Y.W., Q.H. and

588 P.C. analyzed and interpreted the data. C.H.G. and P. C. wrote this paper with the help of all 589 authors. 
591

592

593

594

595

596

597

598

599

600

601

602

603

604

605

606

607

608

609

610

\section{Supplementary materials}

Supplementary Figure S1: Alpha diversity of soil biofilm communities.

Supplementary Figure S2: The biofilm formation of mono-culture of seven soil isolates.

Strains: 1: Aquitalea sp. G2; 2: Novosphingobium sp. G5; 3: Pseudomonas sp. G7; 4:

Flavobacterium sp. G16; 5: Acinetobacter sp. G18; 6: Delftia sp. D10; 7: Microbacterium

sp. D12.

Supplementary Figure S3: Colony inhibitory assays. From left to right and from top to

bottom, 3 microliters of bacterial culture from 7 different isolates were sequentially

inoculated on LB plates (A) or on bacterial lawn of each of the 7 isolates (B-H) (as listed

and ordered by 1-7 in Fig. 4). After 24 hours of cultivation, if the colonies can produce

antibiotics and inhibit the growth of the lawn species, a inhibitory zone will be seen.

Supplementary Figure S4: Alignment of Pseudomonas sp. G7 Unigenes to

Pseudomonas sp. DR 5-09 bananamide biosynthesis gene cluster.

Supplementary Table S1: Bacterial isolates from cultivated biofilm community.

Supplementary Table S2: Biofilms formed by single species and combinations (four

strains).

\section{References}

1. Flemming H-C, Wingender J, Szewzyk U, Steinberg P, Rice SA, Kjelleberg S.

2016. Biofilms: an emergent form of bacterial life. Nat Rev Microbiol 14:563-575.

2. Potera C. 1996. Biofilms invade microbiology. Science 273:1795-1797. 

biofilms: do they actually matter? Trends Microbiol 22:84-91.

5. Hibbing ME, Fuqua C, Parsek MR, Peterson SB. 2010. Bacterial competition:

616 surviving and thriving in the microbial jungle. Nat Rev Microbiol 8:15-25.

6. Szamosvari D, Rütschlin S, Böttcher T. 2018. From pirates and killers: Does

618 metabolite diversity drive bacterial competition? Org Biomol Chem. 
10. Rutledge PJ, Challis GL. 2015. Discovery of microbial natural products by

630 activation of silent biosynthetic gene clusters. Nat Rev Microbiol 13:509-523.

637 discovery of the malacidins as calcium-dependent antibiotics with activity against 638 multidrug-resistant Gram-positive pathogens. Nat Microbiol 3:415-422.

642 Schuemann J, Martin K, Hertweck C, Brakhage AA. 2009. Intimate bacterial-fungal 643 interaction triggers biosynthesis of archetypal polyketides in Aspergillus nidulans. Proc Natl 644 Acad Sci U S A 106:14558-14563. 
647

648

649

650

651

652

653

654

655

656

657

658

659

660

661

662

663

664

665

Rajski SR, Bugni TS. 2017. Coculture of Marine Invertebrate-Associated Bacteria and Interdisciplinary Technologies Enable Biosynthesis and Discovery of a New Antibiotic, Keyicin. ACS Chem Biol 12:3093-3102.

16. Ren D, Madsen JS, Sørensen SJ, Burmølle M. 2015. High prevalence of biofilm synergy among bacterial soil isolates in cocultures indicates bacterial interspecific cooperation. ISME J 9:81-89.

17. Nguyen DD, Melnik AV, Koyama N, Lu X, Schorn M, Fang J, Aguinaldo K, Lincecum TL, Ghequire MGK, Carrion VJ, Cheng TL, Duggan BM, Malone JG, Mauchline TH, Sanchez LM, Kilpatrick AM, Raaijmakers JM, Mot RD, Moore BS, Medema MH, Dorrestein PC. 2016. Indexing the Pseudomonas specialized metabolome enabled the discovery of poaeamide B and the bananamides. Nat Microbiol 2:16197.

18. Medema MH, Kottmann R, Yilmaz P, Cummings M, Biggins JB, Blin K, Bruijn I de, Chooi YH, Claesen J, Coates RC, Cruz-Morales P, Duddela S, Düsterhus S, Edwards DJ, Fewer DP, Garg N, Geiger C, Gomez-Escribano JP, Greule A, Hadjithomas M, Haines AS, Helfrich EJN, Hillwig ML, Ishida K, Jones AC, Jones CS, Jungmann K, Kegler C, Kim HU, Kötter P, Krug D, Masschelein J, Melnik AV, Mantovani SM, Monroe EA, Moore M, Moss N, Nützmann H-W, Pan G, Pati A, Petras D, Reen FJ, Rosconi F, Rui Z, Tian Z, Tobias NJ, Tsunematsu Y, Wiemann P, Wyckoff E, Yan X, Yim G, Yu F, Xie Y, Aigle B, Apel AK, Balibar CJ, Balskus EP, Barona-Gómez F, Bechthold A, Bode HB, Borriss R, 
Tang Y, Truman AW, Viaud M, Walton JD, Walsh CT, Weber T, Wezel GP van, Wilkinson B,

Willey JM, Wohlleben W, Wright GD, Ziemert N, Zhang C, Zotchev SB, Breitling R,

678 Takano E, Glöckner FO. 2015. Minimum Information about a Biosynthetic Gene cluster.

679 Nat Chem Biol. Comments and Opinion.

681 Preston GM, Zhang X-X, Moon CD, Gehrig SM, Godfrey SAC, Knight CG, Malone JG,

682 Robinson Z, Spiers AJ, Harris S, Challis GL, Yaxley AM, Harris D, Seeger K, Murphy L,

683 Rutter S, Squares R, Quail MA, Saunders E, Mavromatis K, Brettin TS, Bentley SD,

684 Hothersall J, Stephens E, Thomas CM, Parkhill J, Levy SB, Rainey PB, Thomson NR. 2009.

685 Genomic and genetic analyses of diversity and plant interactions of Pseudomonas 
20. Vlassak K, Holm LV, Duchateau L, Vanderleyden J, Mot RD. 1992. Isolation

and characterization of fluorescent Pseudomonas associated with the roots of rice and

691 Soil-Microbe Complex. Science 304:1634-1637.

693 functional consequences. J Plant Nutr Soil Sci 180:425-429.

697 Evaluation of microbial biofilm communities from an Alberta oil sands tailings pond.

698 FEMS Microbiol Ecol 79:240-250. Soil. PLOS ONE 9:e87217. 
29. Vos M, Wolf AB, Jennings SJ, Kowalchuk GA. 2013. Micro-scale

719 Truu J. 2014. Characterization of bacterial communities in soil and sediment of a created

720 riverine wetland complex using high-throughput 16S rRNA amplicon sequencing. Ecol Eng 
Co-existence facilitates interspecific biofilm formation in complex microbial communities: 
40. Quast C, Pruesse E, Yilmaz P, Gerken J, Schweer T, Yarza P, Peplies J,

741 Glöckner FO. 2013. The SILVA ribosomal RNA gene database project: improved data

742 processing and web-based tools. Nucleic Acids Res 41:D590-D596.

41. Parks DH, Tyson GW, Hugenholtz P, Beiko RG. 2014. STAMP: statistical

analysis of taxonomic and functional profiles. Bioinformatics 30:3123-3124.

42. Ren D, Madsen JS, de la Cruz-Perera CI, Bergmark L, Sørensen SJ, Burmølle

M. 2014. High-Throughput Screening of Multispecies Biofilm Formation and Quantitative

PCR-Based Assessment of Individual Species Proportions, Useful for Exploring

748 Interspecific Bacterial Interactions. Microb Ecol 68:146-154. 
45. Huson DH, Auch AF, Qi J, Schuster SC. 2007. MEGAN analysis of

759 metagenomic data. Genome Res 17:377-386.

46. Zhu W, Lomsadze A, Borodovsky M. 2010. Ab initio gene identification in

metagenomic sequences. Nucleic Acids Res 38:e132-e132.

47. Li B, Dewey CN. 2011. RSEM: accurate transcript quantification from

RNA-Seq data with or without a reference genome. BMC Bioinformatics 12:323.

48. Langmead B, Salzberg SL. 2012. Fast gapped-read alignment with Bowtie 2.

49. Camacho C, Coulouris G, Avagyan V, Ma N, Papadopoulos J, Bealer K, an online repository of Standard Operating Procedures (SOPs) for (meta)genomic

771 annotation. Omics J Integr Biol 12:137-141.

773 package for multiple sequence alignment. Bioinformatics 31:3997-3999.

774 52. Paradis E, Claude J, Strimmer K. 2004. APE: Analyses of Phylogenetics and 775 Evolution in R language. Bioinformatics 20:289-290. 
777 visualization and annotation of phylogenetic trees with their covariates and other associated 778 data. Methods Ecol Evol 8:28-36. affects the expressions of diverse genes in Mycobacterium smegmatis. Nucleic Acids Res 40:1009-1020. using real-time quantitative PCR and the 2(-Delta Delta C(T)) Method. Methods San Diego

Calif 25:402-408. 

asterisk before the strain name indicates that the strain was selected for further co-cultivation and validation. Details of the strain can be found in Table S1.

\section{Figure 4 The biofilm formation of four-species co-cultures of seven soil isolates}

807 Pseudomonas sp. G7; 4: Flavobacterium sp. G16; 5: Acinetobacter sp. G18; 6: Delftia sp.

808 D10; and 7: Microbacterium sp. D12.

\section{Figure 5 Gene expression changes in four species co-culture}

(A) Volcano plot and MA-plot of differentially expressed genes (DEG). Blue points

811 represent down regulated DEG. Grey points represent non-DEGs. Genes that belong to

812 different species were marked with different shapes as indicated by the Figure legend. (B)

813 Functional analysis of DEGs. Number of DEGs in each species are given in the title of each

814 graph. Numbers of COGs are shown in bar plots, and down- (blue) and upregulated (red) 
817 are shown in the right panel.

818 Figure 6 Novel antibiotic biosynthesis gene cluster and predicted molecular structure the compound is shown on above.

Figure 7 Comparative analysis of the paddyleftmide and paddyrightmide BGC with

828 Maximum likelihood phylogenetic tree (A, C) was inferred by multilocus sequence analysis

829 of core synthetic genes and the pairwise phylogenetic distance between them $(\mathrm{B}, \mathrm{D})$ were

830 shown in heat map with same color theme.

831 Figure 8 The induction of NRPS synthetic gene in co-culture revealed by qRT-PCR

832 assays.

The induction of paddyleftmide (A) and paddyrightmide (B) synthetic gene in

834 Pseudomonas sp. G7 monoculture, two-, three- and four-species co-culture were showed. 
835 Gene expression was normalized by $16 \mathrm{~S}$ rRNA gene of Pseudomonas sp. G7, and changes

836 were calculated by the $2^{-\Delta \Delta \mathrm{Ct}}$ method. Errorbars represent standard deviations of three

837 replicates. The species contained in the co-cultivation show as numbers in horizontal axis,

838 and the numbers corresponding to the species are the same as in Fig. 4.

839

840 
841 Table 1 Gene expression changes of NRPS BGCs

\begin{tabular}{lrrrll}
\hline Meta GeneID & FC & log2FC & P-value & WGS locus_tag & NRPS cluster \\
\hline Pseudgene_4428 & 35.6 & 5.2 & 0 & C6Y56_11445 & Paddyleftmide BGC \\
Pseudgene_4429 & 27.0 & 4.8 & 0 & C6Y56_11450 & Paddyleftmide BGC \\
Pseudgene_5485 & 66.5 & 6.1 & 0 & C6Y56_11445 & Paddyleftmide BGC \\
Pseudgene_5486 & 27.0 & 4.8 & 0 & C6Y56_11450 & Paddyleftmide BGC \\
Pseudgene_5487 & 24.7 & 4.6 & 0 & C6Y56_11445 & Paddyleftmide BGC \\
Pseudgene_5488 & 16.1 & 4.0 & $2.16 \mathrm{E}-14$ & C6Y56_11445 & Paddyleftmide BGC \\
Pseudgene_5489 & 27.0 & 4.8 & 0 & C6Y56_11450 & Paddyleftmide BGC \\
Pseudgene_6748 & 27.0 & 4.8 & 0 & C6Y56_11445 & Paddyleftmide BGC \\
Pseudgene_6749 & 33.5 & 5.1 & 0 & C6Y56_11450 & Paddyleftmide BGC \\
Pseudgene_6750 & 26.2 & 4.7 & 0 & C6Y56_11440 & Paddyleftmide BGC \\
Pseudgene_6751 & 35.6 & 5.2 & 0 & C6Y56_11440 & Paddyleftmide BGC \\
Pseudgene_6780 & 24.0 & 4.6 & 1.11E-16 & C6Y56_11440 & Paddyleftmide BGC \\
Pseudgene_303 & 9.1 & 3.2 & $4.09 \mathrm{E}-10$ & C6Y56_11685 & Paddyrightmide BGC \\
Pseudgene_304 & 4.6 & 2.2 & 4.96E-05 & C6Y56_11680 & Paddyrightmide BGC \\
\hline
\end{tabular}

842 Note: Meta GeneID is the coding system that used in Meta-transcriptomic analysis. The

843 gene ID in the maintext has been replaced by the formal locus_tag.

844 
Table 2 The genome and general information of related Pseudomonas spp.

\begin{tabular}{|l|l|l|l|lr|}
\hline Strain & Accession & Location & Date & Reference & \\
\hline $\begin{array}{l}\text { Pseudomonas sp. } \\
\text { DR 5-09 }\end{array}$ & CP011566 & $\begin{array}{l}\text { Daecheong, } \\
\text { South Korea. } \\
\text { Plant root. }\end{array}$ & $\begin{array}{l}\text { Sep, } \\
\text { 2012. }\end{array}$ & $\begin{array}{l}\text { Han,J.H. and Kim,S.B. } \\
\text { Unpublished. }\end{array}$ \\
\hline $\begin{array}{l}\text { Pseudomonas sp. } \\
\text { MS586 }\end{array}$ & CP014205 & $\begin{array}{l}\text { Cotton field, } \\
\text { USA }\end{array}$ & $\begin{array}{l}\text { Aug, } \\
2012\end{array}$ & $\begin{array}{l}\text { Lu,S. and } \\
\text { Unpublished. }\end{array}$ & Deng,P. \\
\hline $\begin{array}{l}\text { Pseudomonas } \\
\text { moraviensis strain } \\
\text { BS3668 }\end{array}$ & LT629788 & NA & $\begin{array}{l}\text { Feb, } \\
2016\end{array}$ & $\begin{array}{l}\text { Varghese,N. } \\
\text { Submissions,S. } \\
\text { Unpublished. }\end{array}$ & \\
\hline $\begin{array}{l}\text { Pseudomonas } \\
\text { fluorescens Pf0-1 }\end{array}$ & CP000094 & $\begin{array}{l}\text { Agricultural } \\
\text { loam soil }\end{array}$ & 1988 & (19) \\
\hline $\begin{array}{l}\text { Pseudomonas } \\
\text { fluorescens strain } \\
\text { BW11P2 }\end{array}$ & KX437753 & $\begin{array}{l}\text { Banana } \\
\text { rhizoplane, Sri } \\
\text { Lanka }\end{array}$ & $\begin{array}{l}\text { Paddy soil, } \\
\text { China }\end{array}$ & 2016 & This study. \\
\hline $\begin{array}{l}\text { Pseudomonas sp. } \\
\text { G7 }\end{array}$ & CP027561 & & \\
\hline
\end{tabular}




\section{Isolation}

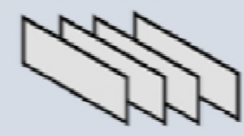

Screening

\section{Targeting}

\section{Verification}

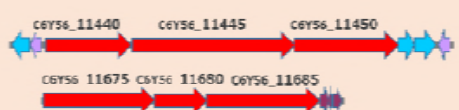

whole genome sequencing
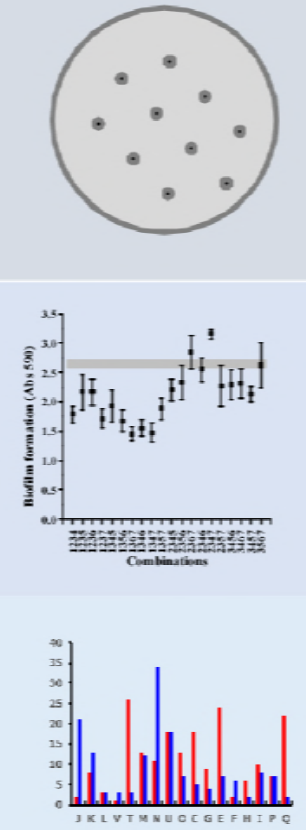

cultivate biofilm in agar-coated microslide

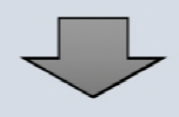

isolate cultivable bacterial species

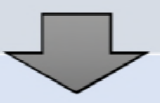

multispecies biofilm formation using a co-culture system

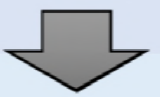

meta-transcriptomics

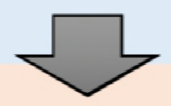


A

$100 \%$
$80 \%$
$60 \%$
$40 \%$
$20 \%$
$0 \%$
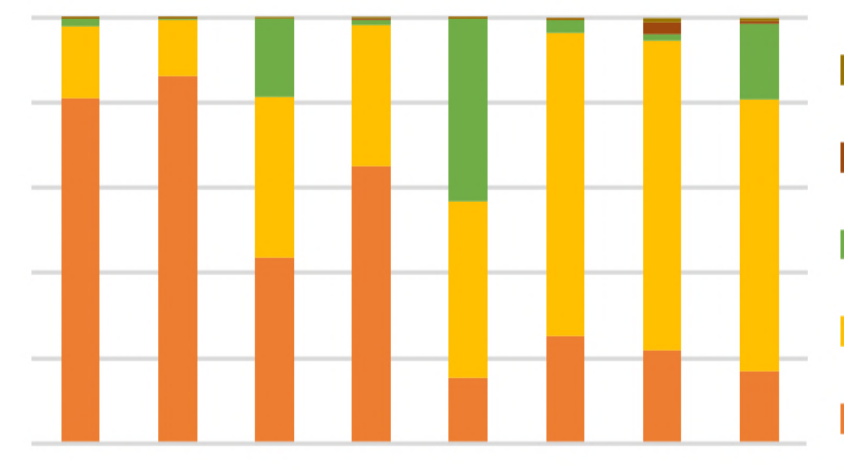

Others

- Verrucomicrobia

- Bacteroidetes

Proteobacteria

Firmicutes

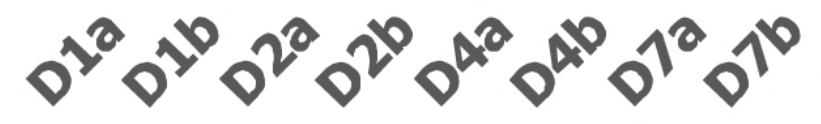

B

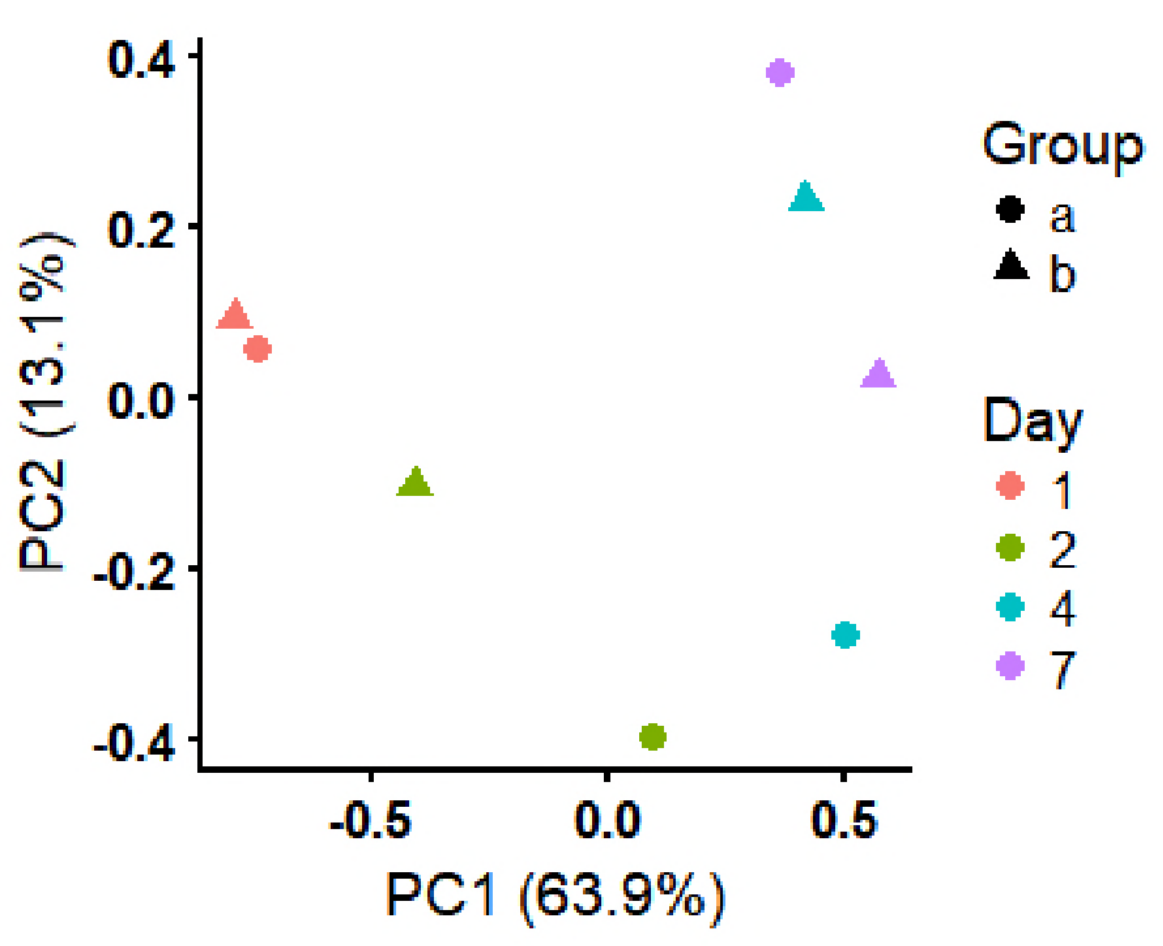

C

Firmicutes

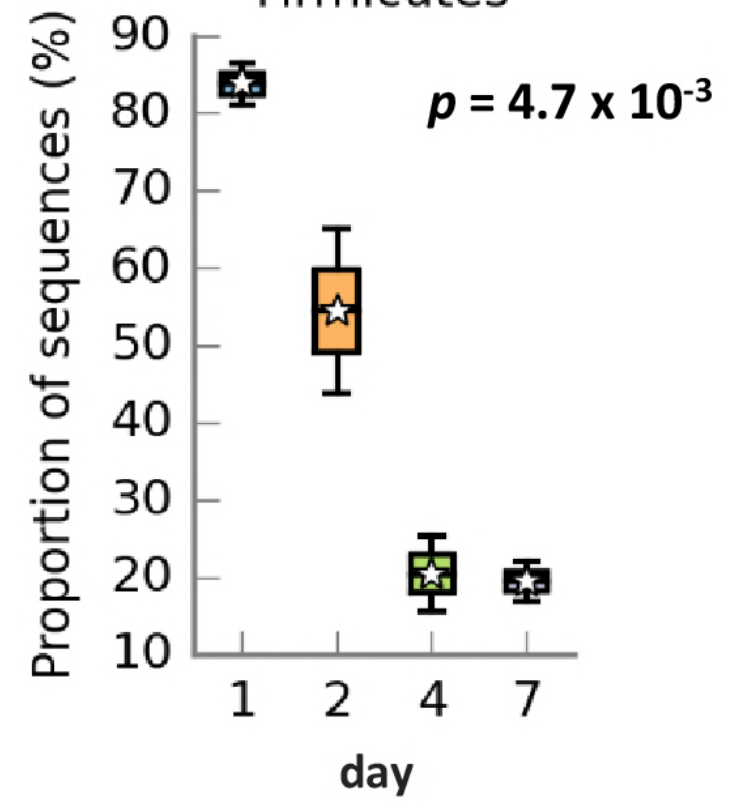

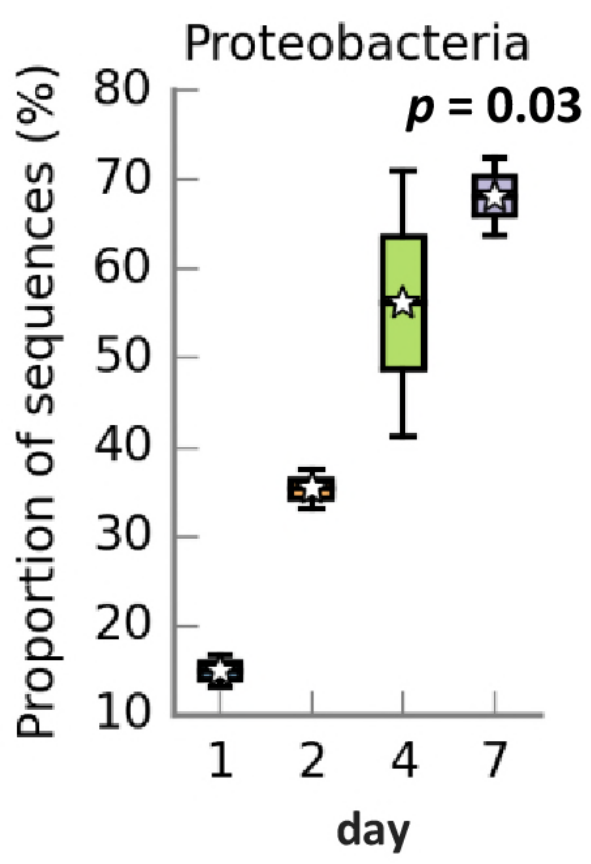




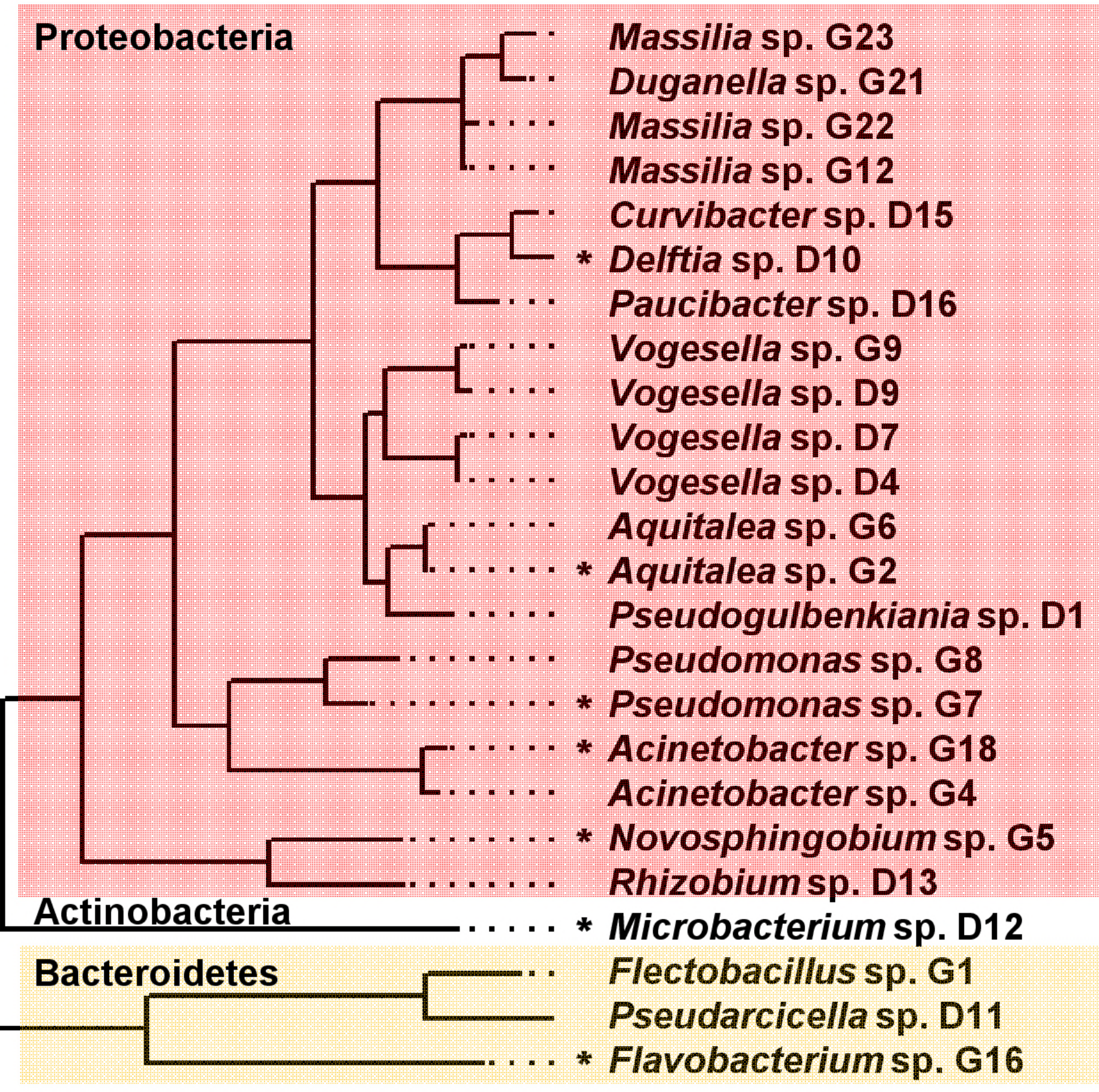


A $\quad$ B
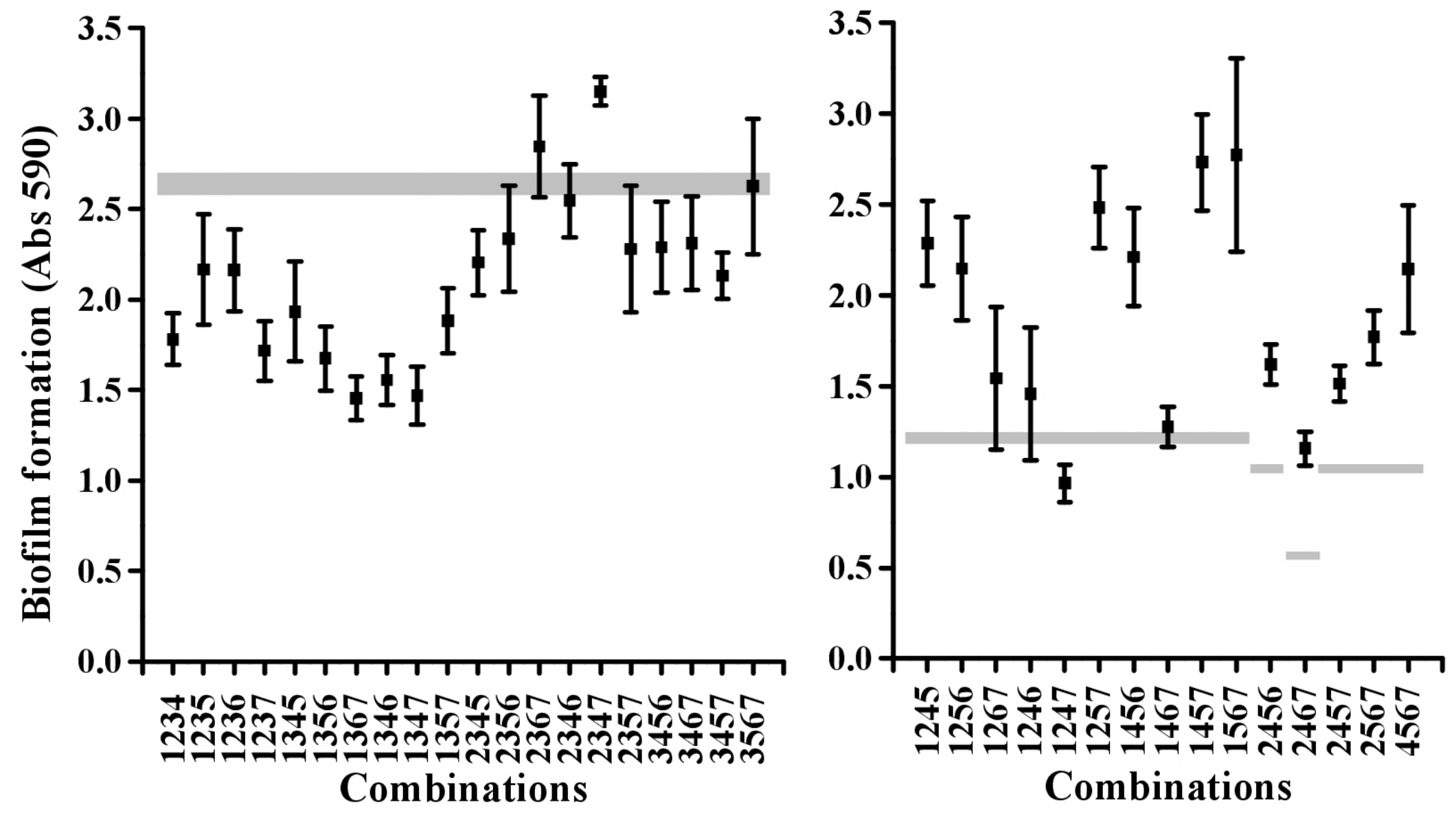


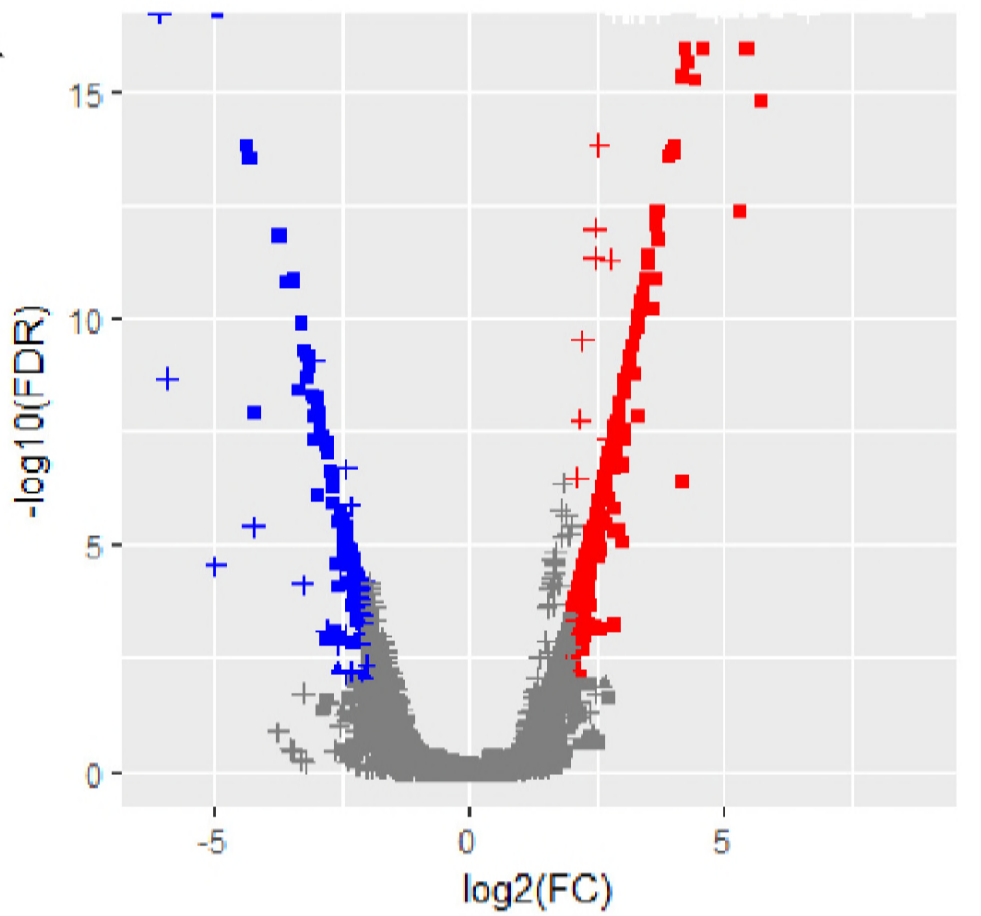

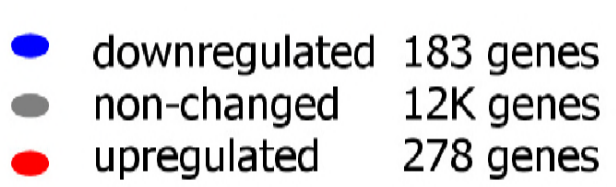

B

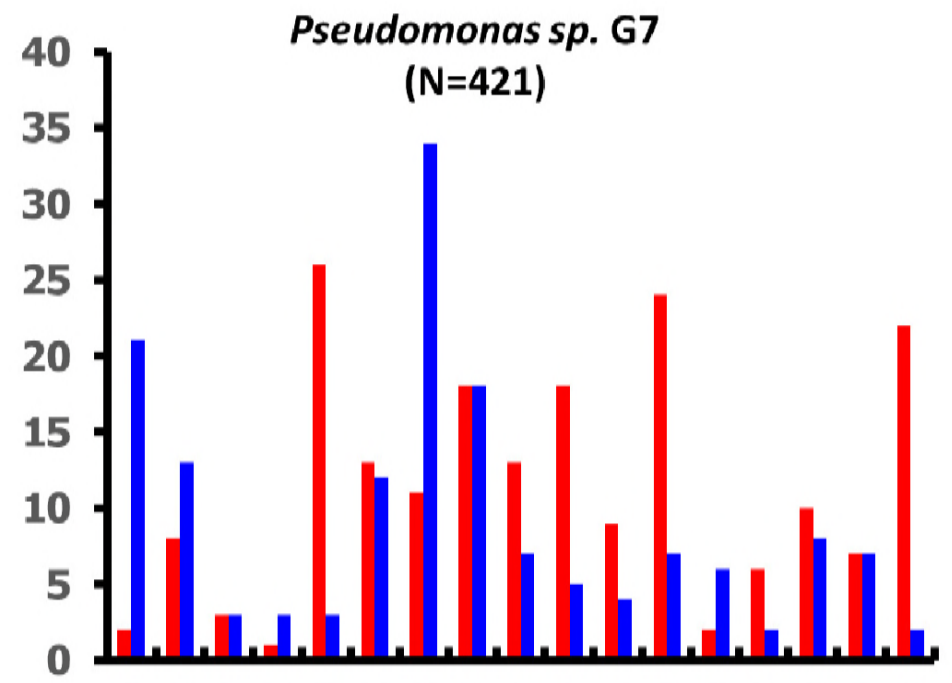

J K L V T M N U O C G E F H I P Q

Novosphingomonas sp. G5

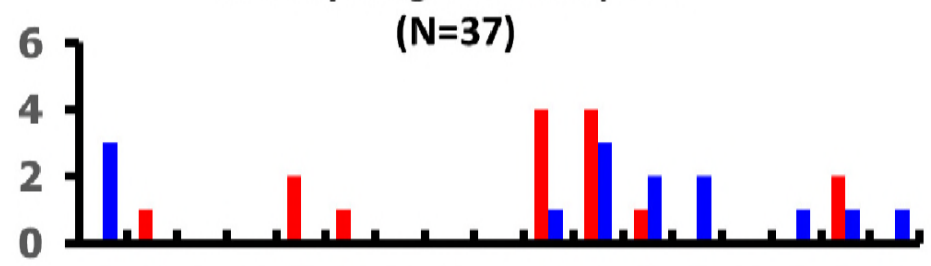

J K L V T M N U O C G E F H I P Q Microbacterium sp. D12

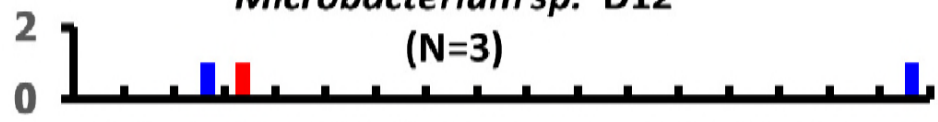

J K L V T M N U O C G E F H I P Q

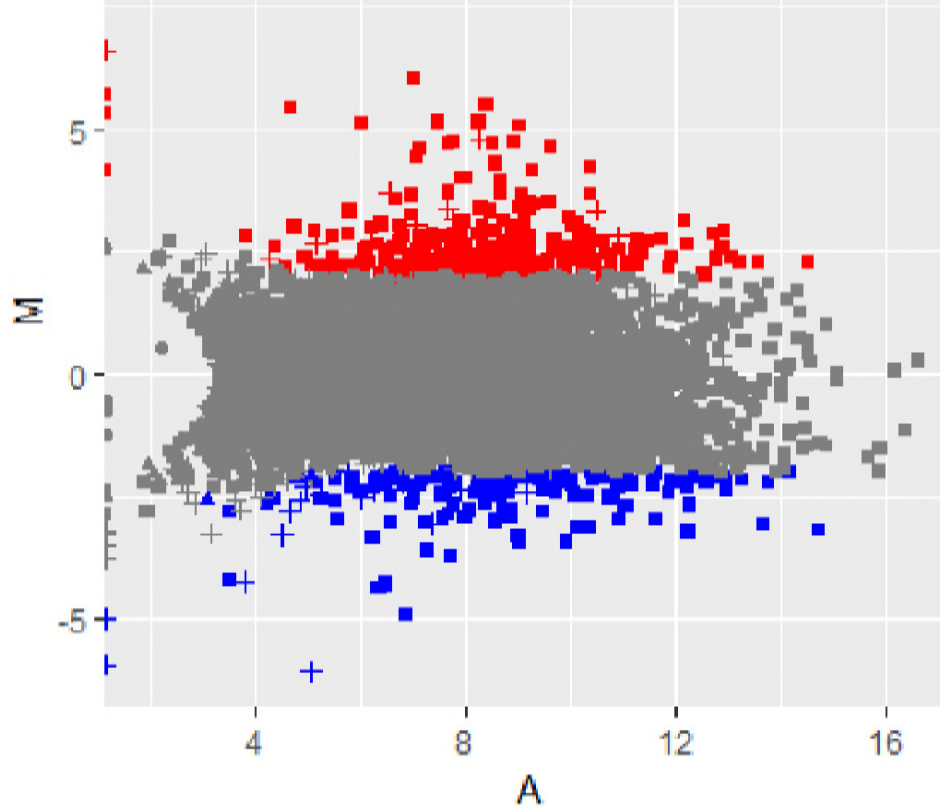

+ Novosphingobiumsp. G5 37 genes

- Pseudomonas sp. G7 421 genes

- Flavobacteriumsp. G16 0 genes

- Microbacterium sp. D12 3 genes

\begin{tabular}{cl}
\hline Code & \multicolumn{1}{c}{ Functional.Categories } \\
\hline J & $\begin{array}{l}\text { Translation, ribosomal structure and } \\
\text { biogenesis }\end{array}$ \\
K & Transcription \\
L & Replication, recombination and repair \\
V & Defense mechanisms \\
T & Signal transduction mechanisms \\
M & Cell wall/membrane/envelope biogenesis \\
N & Cell motility \\
U & Intracellular trafficking, secretion, and \\
& vesicular transport \\
O & Posttranslational modification, protein \\
C & turnover, chaperones \\
G & Carbohydrate transport and metabolism \\
E & Amino acid transport and metabolism \\
F & Nucleotide transport and metabolism \\
H & Coenzyme transport and metabolism \\
I & Lipid transport and metabolism \\
P & Inorganic ion transport and metabolism \\
Q & Secondary metabolites biosynthesis, transport \\
\hline
\end{tabular}


A

$35 \mathrm{~kb}$

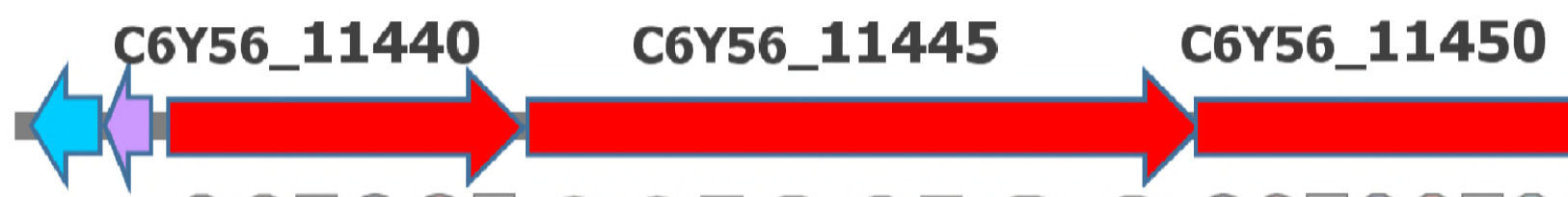

C.ATCAT C A T C A T C A CATCAT TETE

\section{B}

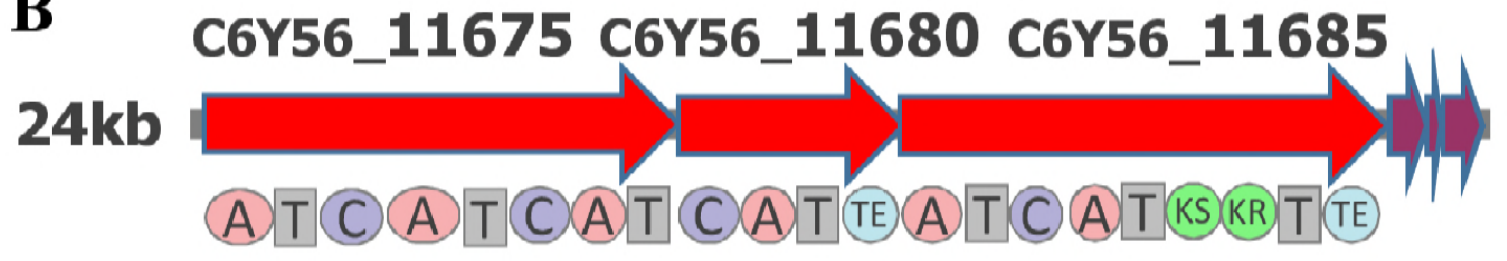

Core biosynthetic genes

$\square$ Transport-related genes

$\square$ Regulatory genes

Hypothetical proteins

C) Codensation (A)Adenylation T Thiolation (TE)Thioesterase ${ }^{K S} \beta$-ketoacyl synthase KR ketoreductase

C<smiles>CCC(C)[C@H](C=O)NC(=O)[C@H](CC(C)C)NC(=O)[C@H](CC(C)C)NC(=O)[C@H](NC(=O)[C@H](NC(=O)[C@H](CC(=O)O)NC(=O)[C@@H](N)CC(C)C)C(C)O)C(C)O</smiles>

D

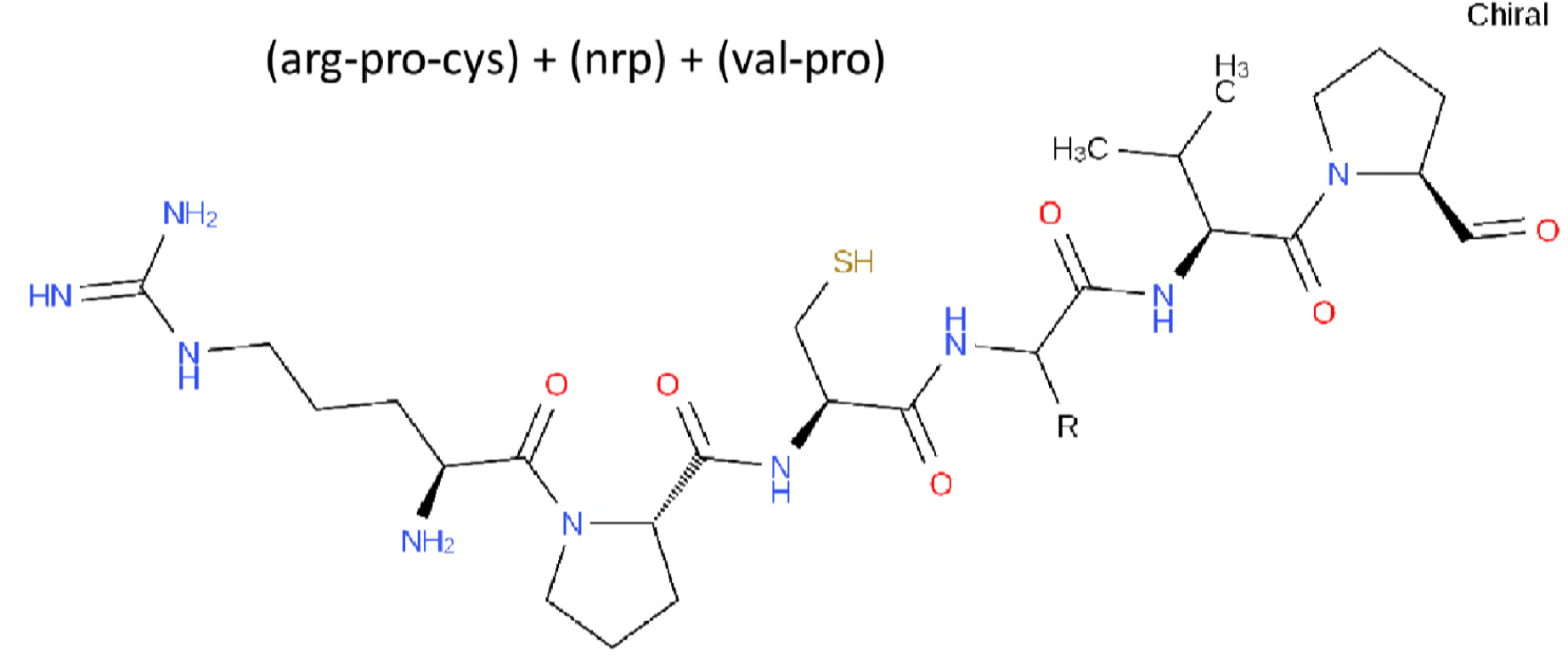




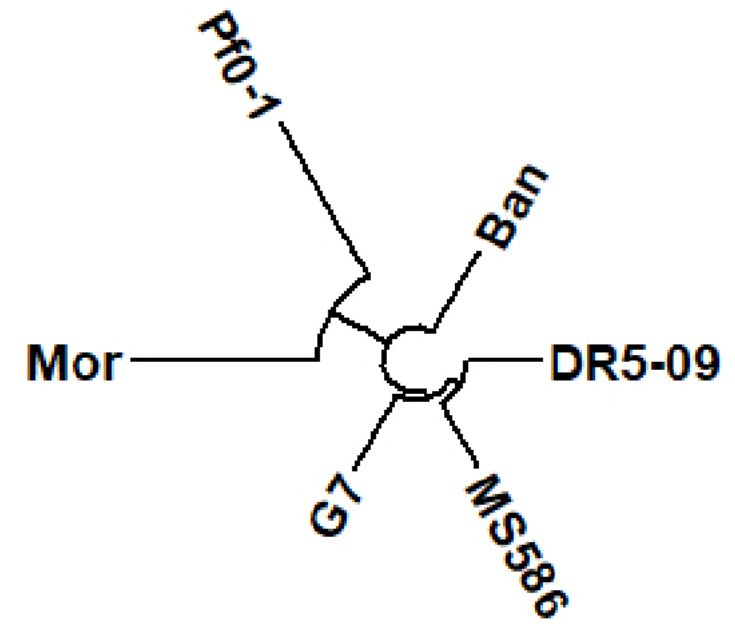

C

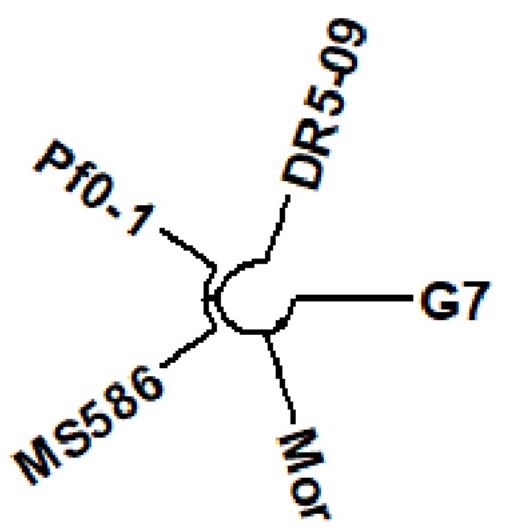

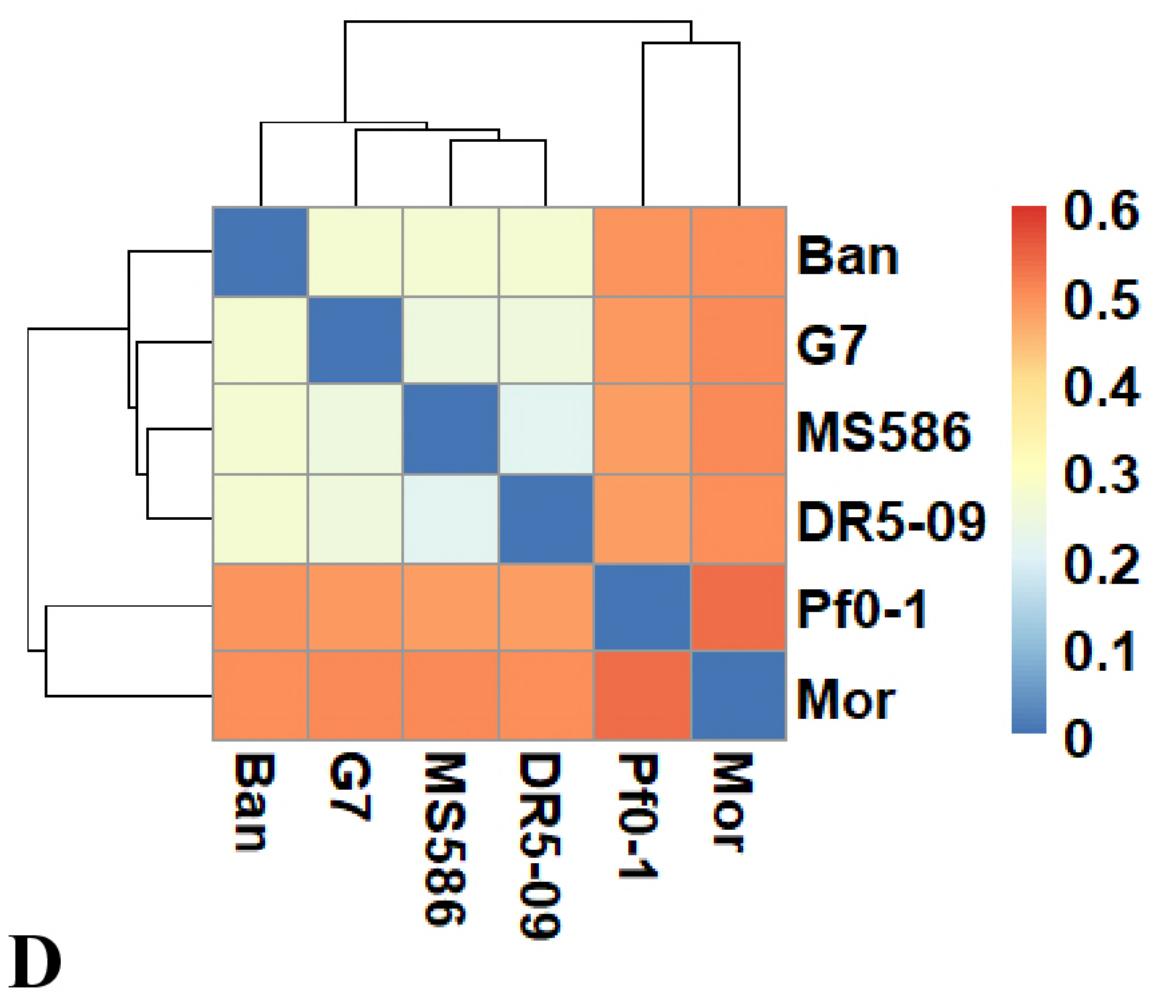

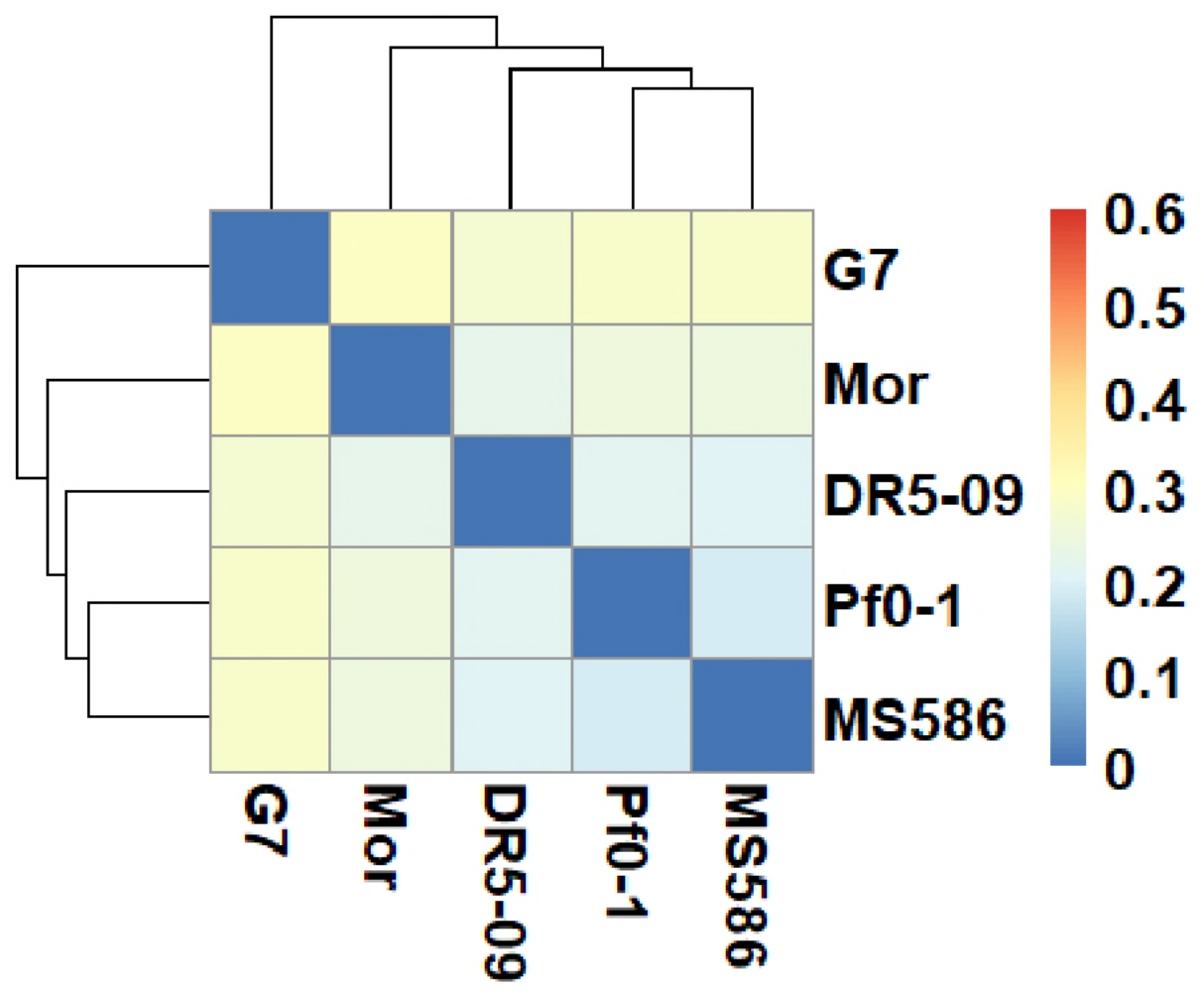


A 2 Paddyleftmide synthetic gene C6Y56_11440

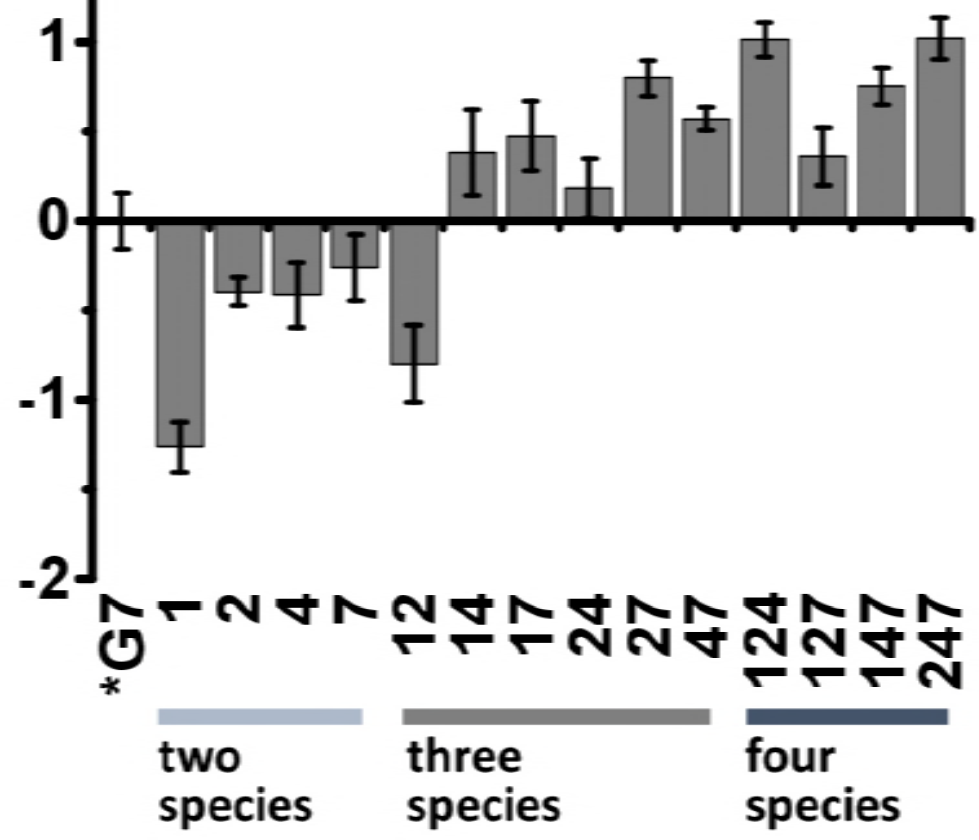

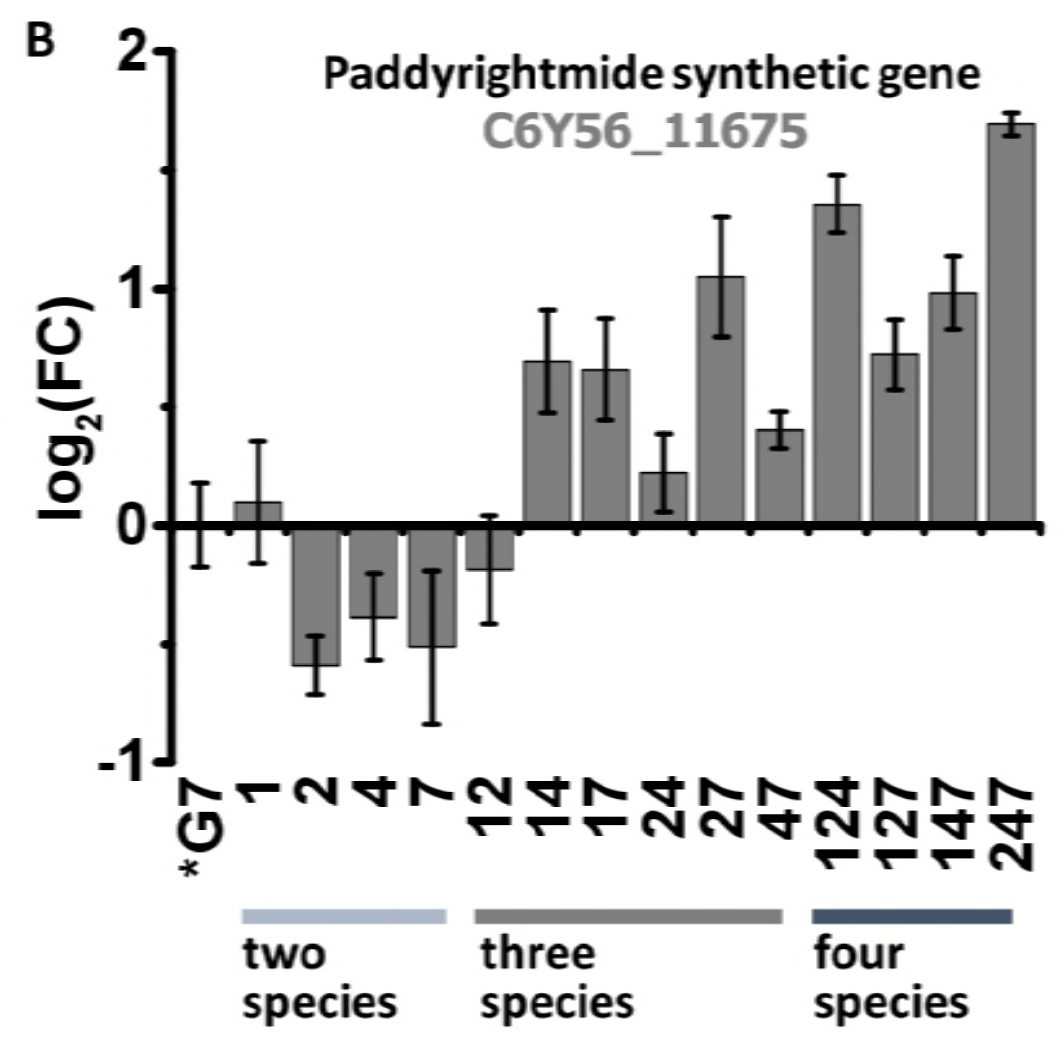

